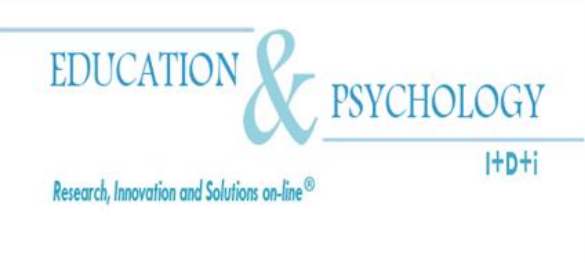

\title{
Ayudas al aprendizaje en tareas de escritura colaborativa con Moodle
}

\author{
Teresa Mauri, Rosa M. Colomina, Marc Clarà, Anna Ginesta
}

Dpto. de Psicología Evolutiva y de la Educación.

Universidad de Barcelona

\section{España}

Correspondencia: Teresa Mauri Majós. Pg. de la Vall d'Hebrón, 171. 08035, Barcelona. España tmauri@ub.edu

(C) Education \& Psychology I+D+i and Editorial EOS (Spain) 


\section{Resumen}

Introducción. La incorporación de las TIC a la docencia ha modificado las condiciones en que los alumnos que colaboran en la elaboración de un texto logran aprender. En consecuencia, también se han transformado las opciones de los profesores para prestar ayuda educativa en este proceso. Por todo ello, en este escrito queremos explorar las características que toma dicha ayuda en este tipo de tareas. Más en concreto, pretendemos conocer los aspectos de la tarea a los que se da ayuda; los momentos en que ésta se presta y, dados los cambios, identificar algunos criterios que deberían orientar las actuaciones docentes en esta tarea en la actualidad. Esta investigación se fundamenta en la perspectiva socioconstructivista de los procesos de enseñanza y aprendizaje y ha sido llevada a cabo con apoyo de la plataforma Moodle.

Método. Con una metodología cualitativa (grounded theory) y de estudio de casos, se han registrado y analizado datos de dos casos cuyos participantes son dos profesoras y cincuenta alumnos trabajando en grupos de 4-5 estudiantes en una secuencia didáctica de la asignatura de Psicologia de la Educación de primer curso de la formación de maestros. Además del registro audiovisual y electrónico de los datos se han realizado entrevistas con profesoras y alumnos. Se elaboró un protocolo de análisis (dimensiones, subdimensiones e indicadores) de las potencialidades y limitaciones de la propuesta docente, las ayudas ofrecidas, y los resultados de aprendizaje.

Resultados. Los estudiantes reciben ayudas educativas para mejorar la gestión de su participación, de la tarea y de los significados. Sin embargo estas ayudas no se dirigen por igual a cada una de estas dimensiones, siendo la gestión de la tarea la más apoyada. Además existen diferencias en relación a cuándo (al inicio, durante y al final del proceso) y a sobre qué se proporcionan las ayudas, así como en el uso mediador de las TIC en el aprendizaje.

Discusión y Conclusión. El estudio muestra la importancia de ayudar a los estudiantes a gestionar la participación, la tarea y la negociación del significado compartido para aprender juntos. También evidencia la necesidad de incrementar el número y la calidad de las ayudas fundamentalmente durante el proceso de la tarea y, en especial, de las que se prestan a la gestión de la construcción del significado.

Palabras Clave: Aprendizaje en Educación Superior; Escritura colaborativa online; Actividad conjunta; Ayuda educativa. 


\title{
Support for learning in collaborative writing tasks with Moodle
}

\begin{abstract}
Introduction. The incorporation of ICT in teaching process has changed the conditions in which students learn when collaboratively writing a text. In consequence, in this process, the way in which teachers can offer support has also changed. For this reason, in this paper we would like to explore the characteristics and the forms of this kind of in these specific tasks. Concretely, we would like to know the aspects of the task in which the support is addressed; the moments in which is offered and identify some criteria that have actually helped to orientate the teacher actions in these tasks. This research is based on a socioconstructivist perspective of the teaching and learning processes, and is has been put in to effect with the support of the Moodle platform.

Method. With a qualitative methodology (grounded theory) and case studies, we have recorded and analysed data from two different cases. The participants are two teachers and fifty students working in groups of four or five in a didactic sequence within the subject of Psychology of Education from the first course of the degree on Teacher Education. As well as this, audiovisual and electronic register of the data, we have interviewed the teachers and the students. We elaborated a protocol of analysis (dimensions, subdimensions and indicators) of the potentials and limitations of the teacher plan, the help offered, and the learning results.

Results: The students receive educational assistance to improve the management of their participation, the management of the task and the management of the meanings. Although, this help they don't face them equally. Task management is the most supported dimension by the teacher. Furthermore, there exists differences in relation with when (at the beginning, during and after the process) and on what the help is offered, there also exist differences on the mediating use of ICT in the learning process.

Discussion and Conclusion: The study shows the importance of helping students to manage the collaboration, the task and the process of co-negotiate meanings to learn together. At the same time, the study shows evidence of the necessity to increase the amount and the quality of help, fundamentally, during the process of the task, and especially, the assistance addressed to manage the construction of meanings.
\end{abstract}

Keywords: Learning in higher education. Online collaborative writing. Joint activity. Educational assistance. 


\section{Introducción}

Una de las tareas habituales de los estudiantes en la universidad es la elaboración de textos escritos en grupo con la finalidad de aprender y mostrar lo que han aprendido. En los últimos años dicha tarea se realiza en muchas universidades españolas mediada por la plataforma Moodle, por lo que profesores y alumnos disponen de herramientas virtuales de apoyo al proceso de enseñanza y aprendizaje. Entre los recursos que la plataforma incorpora se usa con frecuencia el "forum" para trabajar en pequeño grupo. Dicho espacio virtual, que tecnológicamente no ha sido creado específicamente para apoyar este tipo de tareas, permite en cada contribución el envío de mensajes y de textos adjuntos. Mientras el espacio del forum sirve para la comunicación entre estudiantes sobre temáticas muy variadas, el envío de adjuntos sirve para intercambiar las sucesivas versiones del texto que los miembros del grupo elaboran y se remiten entre sí hasta componer conjuntamente la versión final del mismo. También es posible en ese espacio la intervención del profesor de ayuda a la colaboración para la construcción conjunta del conocimiento. La incorporación de las TIC a la docencia ha modificado las características de esta tarea y también las posibilidades de profesores y alumnos de prestar y recibir ayuda educativa, por lo que actualmente conocemos muy poco de las exigencias que la realización de la misma les plantea. En consecuencia parece necesario profundizar en el estudio de estas características y de su real contribución al aprendizaje.

En este trabajo se aborda el estudio de las formas de ayuda educativa del profesor al aprendizaje de contenidos académicos por los alumnos mediante su implicación en tareas de escritura colaborativa de textos con el forum de la plataforma Moodle. La finalidad es conocer la influencia educativa que el profesor realiza en dicha tarea con esta herramienta para que los alumnos aprendan, y obtener elementos de reflexión para contribuir a mejorarla.

El principal referente teórico de este estudio es la perspectiva constructivista sociocultural del aprendizaje y la enseñanza. El aprendizaje es entendido como un proceso de construcción personal y conjunta del conocimiento por el que los alumnos elaboran el significado de los contenidos y las tareas de aprendizaje y les atribuyen sentido. De acuerdo con los planteamientos de Vygotsky $(1978,1986)$ y Wells (1999), se trata de un proceso mediado por la asistencia o ayuda educativa del profesor, o de los demás alumnos del grupo de trabajo, que comporta la creación de una Zona de Desarrollo Próximo (ZDP) en la que toman sentido di- 
cha asistencia y construcción del significado. La importancia atribuida a la ayuda educativa y, en particular al ajuste continuado de dicha ayuda para lograr la comprensión de los procesos de enseñanza y aprendizaje, lleva a convertir la actividad conjunta de profesor y alumnos entorno a los contenidos en la unidad principal de estudio de la influencia educativa. En consecuencia con ello, abordar la actividad conjunta supone abordar un doble proceso de construcción. Por una parte el proceso de construcción de la estructura de participación, que se configura a su vez, en la elaboración de la estructura de participación social (quién puede decir o hacer qué, cuando, cómo, dirigiéndose a quién y con qué medios) y de la estructura de la tarea académica (qué hay que hacer, cómo hay que hacerlo, mediante qué procedimientos, que productos hay que generar, con qué características). Por otra parte, supone abordar la construcción conjunta de significados compartidos; es decir, el proceso por el que los significados son comprendidos, negociados y compartidos por los participantes. Todos estos procesos tienen lugar fundamentalmente mediante el discurso (Coll, 2001).

La relevancia educativa de la tarea de escritura colaborativa en la docencia universitaria se basa no sólo en la calidad de la actividad conjunta de profesores y alumnos, sino también en las potencialidades educativas que se atribuyen a la interacción entre alumnos: a la actividad que desarrollan cuando trabajan conjuntamente para escribir un texto en condiciones de comunicación asíncrona. La complejidad de este proceso ha empezado a ponerse de manifiesto y ha abordarse en estudios como los de Castelló (2009), Castelló, Iñesta y Monereo (2009), Castelló, Bañales y Vega (2010), Cerrato-Pargman (2003), Lowry, Curtis y Lowry (2004), Onrubia y Engel (2009), Saunders (1989) o Van Drie, Van Boxtel, Jaspers y Kanselaar (2005). Todos estos autores subrayan que las potencialidades educativas de la interacción entre alumnos no se manifiestan de forma espontánea por el mero hecho de poner a los alumnos a trabajar juntos y ofrecerles posibilidades de comunicación adecuados. Es decir, para que realmente la colaboración entre alumnos resulte efectiva para el aprendizaje y la construcción conjunta del conocimiento resulta necesaria la existencia de ayudas educativas explícitas y directas del profesor. Ayudas que, de acuerdo con las bases teóricas de este estudio, irán dirigidas a lograr: (i) una adecuada gestión de la participación en la tarea por los estudiantes; (ii) una adecuada gestión de la tarea en sí misma, y (iii) una adecuada gestión de la elaboración conjunta de los significados sobre los contenidos de aprendizaje (Coll, Engel y Bustos, 2009). Que la dispensación de ayuda educativa es una actividad altamente compleja se conoce no sólo porque se debe llevar a cabo en diferentes niveles y momentos del proceso educativo y sobre diferentes aspectos de la actividad conjunta -sobre los contenidos de aprendizaje, la 
concreción de la tarea, la organización y el funcionamiento del grupo, y los recursos tecnológicos- sino también porque dicha ayuda no sirve por sí misma para el aprendizaje, sino que es útil sólo si se lleva a cabo de manera ajustada.

Otro aspecto que otorga una potencial relevancia educativa a la tarea de escritura colaborativa para la construcción del conocimiento es que la escritura puede favorecer la reflexión individual y conjunta para el aprendizaje de los contenidos. Es decir, gracias al uso que los alumnos hacen del discurso escrito, pueden hacer avanzar conjuntamente y de forma cualitativa los significados sobre el contenido (Mauri, Clarà y Remesal, 2011).

El uso de la plataforma Moodle y, en concreto, de la herramienta del forum facilita: (i) que la tarea de escritura colaborativa pueda realizarse de modo asíncrono; (ii) que profesores y estudiantes puedan usar de forma multidireccional la escritura como medio de comunicación; y (iii) que la construcción del conocimiento se pueda llevar a cabo de manera conjunta sosteniendo el acceso, el seguimiento y la elaboración de dichos textos por todos los participantes. Sin embargo, como ya expusimos en anteriores trabajos (Coll, Mauri y Onrubia, 2008a y 2008b), el aprovechamiento de las potencialidades de las TIC para el aprendizaje de contenidos por la implicación de los alumnos en la tarea de escritura colaborativa no depende tan directamente de las posibilidades que abren las herramientas tecnológicas, sino de los usos educativos de las mismas en el proceso de enseñanza y aprendizaje $y$, fundamentalmente, para prestar ayuda. En la concreción del uso de las TIC en la docencia intervienen un extenso campo de decisiones y transformaciones, que abarcan desde las que tomamos sobre las herramientas tecnológicas de la propuesta educativa hasta las prácticas de uso de las mismas con fines educativos en las aulas universitarias. En primer lugar, cabe considerar la toma de decisiones en el nivel del diseño docente, en cuya configuración intervienen tanto las características genéricas de las herramientas tecnológicas como las decisiones pedagógicas sobre sus potenciales usos englobados en una propuesta de conjunto presidida por decisiones educativas. En segundo lugar cabe considerar la redefinición de los usos previstos en la actividad conjunta de profesores y alumnos en el aula al concretarse en este plano los usos reales de las TIC. Por lo tanto, que las posibilidades que abren las TIC tengan efectos transformadores de la enseñanza y el aprendizaje no se deriva directamente de que el apoyo de la comunicación y la colaboración sean tecnológicamente viables, sino que dependen de la existencia de usos educativos de las TIC, previstos en el diseño tecno-pedagógico de la propuesta docente, que hagan posible pedir ayudas educativas y recibirlas. Dichos usos condicionan la naturaleza y la inten- 
sidad de la ayuda pedagógica y se relacionan con el proceso (etapas, fases, momentos) de construcción colaborativa del conocimiento desarrollado por los estudiantes.

Pero, por lo que conocemos actualmente, los usos más frecuentes de las TIC en la docencia universitaria tienen que ver con la búsqueda y el procesamiento de información y los menos frecuentes con la comunicación y la colaboración o, lo que es lo mismo, con su uso en la actividad conjunta como instrumentos de pensamiento entendido como actividad social o “interthinking" (Mercer, 2000). Los usos necesarios para el aprendizaje no ocurren con frecuencia ni espontáneamente entre los alumnos, sino que necesitan de la intervención educativa del profesor quién deberá prestar ayudas dirigidas a lograr la aparición de formas óptimas de construcción colaborativa del conocimiento mediante la escritura de textos on-line.

\section{Objetivos de la investigación}

Nuestro estudio sobre las ayudas educativas ha tomado en consideración el nivel en el que éstas se deciden -el diseño- y aquél en el que se prestan -el desarrollo de la actividad conjunta-. También ha indagado el grado de concreción de esas ayudas y su aparición en diferentes momentos del proceso. Dado que los resultados sobre las ayudas en el nivel del diseño se han presentado en otro escrito (Mauri, Colomina y Ginesta, 2011), en este texto nos centramos en el nivel de la actividad conjunta y el análisis está dirigido a conocer cómo los profesores ejercen la influencia educativa en la práctica. Este trabajo se propone el objetivo de identificar y analizar las características de la ayuda educativa del docente universitario en el desarrollo de tareas de escritura colaborativa de un texto en las que los estudiantes utilizan para el trabajo en pequeño grupo el forum de la plataforma Moodle. Más en concreto, el estudio pretende responder a la pregunta de investigación sobre ¿Cómo los profesores proporcionan ayuda educativa en el proceso de elaboración colaborativa del texto escrito, en cada una de las dimensiones siguientes: gestión de la participación, de la tarea y del significado?

\section{Método}

\section{Contexto y participantes}

Se estudiaron dos casos: dos profesoras y sus grupos clase respectivos de primer curso (G1 y G2), de dos titulaciones de los estudios de Formación del Profesorado (Educación Primaria y Educación Infantil) en la Universidad de Barcelona, que cursaban la asignatura de 
Psicología de la Educación con el uso de la plataforma Moodle. Dicha asignatura es troncal, anual de 12 créditos ECTs y está estructurada en seis secuencias didácticas (SD) ${ }^{1}$. El estudio se centró en la tercera secuencia didáctica: "El aprendizaje escolar. Atribución de sentido". En el G1 seguimos el trabajo de 5 grupos de alumnos, 4 de cinco alumnos y 1 de tres, que colaboraron a lo largo de siete semanas. En el G2 seguimos el trabajo de 5 grupos, 3 de cinco alumnos y 2 de cuatro alumnos, que también colaboraron a lo largo de siete semanas. Los diferentes grupos fueron organizados por los propios alumnos.

La plataforma Moodle fue usada en ambos cursos e incluyó los espacios y herramientas virtuales siguientes: a) un primer espacio donde se depositaban archivos de diferentes tipos con la información necesaria para que los alumnos pudieran gestionar convenientemente su actividad de aprendizaje: guía didáctica, agenda de trabajo del bloque, lecturas y pautas de lecturas, y presentación (powerpoint) de los contenidos del curso; b) un segundo espacio de foro de grupos para la comunicación entre alumnos y con la profesora; c) un tercer espacio de presentación amplia de las actividades; y d) un cuarto espacio con documentos para la autoevaluación individual y conjunta del trabajo realizado y también para la revisión y valoración de los resultados de aprendizaje por parte del profesor. Los materiales docentes fueron elaborados conjuntamente por el equipo de profesores de la asignatura, aunque cada uno los adaptó teniendo en cuenta las características de su propio grupo clase y su estilo de enseñanza.

La tarea de aprendizaje, objeto de estudio, consistía en colaborar a distancia para escribir colaborativamente un texto explicativo que respondiera de manera fundamentada a la pregunta “¿Qué hace que los alumnos atribuyan sentido al aprendizaje?” Todos los estudiantes participaban por primera vez en actividades virtuales en este curso, pero tuvieron la oportunidad de familiarizarse con la plataforma y el uso del foro participando en las actividades desarrolladas en los dos bloques iniciales del curso.

\section{Instrumentos}

Se llevaron a cabo entrevistas a las profesoras al inicio de la secuencia didáctica y al final de la misma. También se recogieron las anotaciones de los diarios elaborados por las

\footnotetext{
${ }^{1}$ Secuencia Didáctica: unidad global de enseñanza y aprendizaje con sentido educativo en sí misma, y que contiene todos los elementos característicos: objetivos, contenidos, actividades de enseñanza y aprendizaje y actividades de evaluación.
} 
profesoras sobre la actividad docente durante el desarrollo de la misma. En relación a los alumnos, se llevó a cabo una entrevista grupal a los estudiantes miembros de los diferentes grupos. Las actuaciones de cada profesora con los alumnos de la muestra en el aula presencial y en las tutorías presenciales fueron registradas en video y audio. Además se registraron electrónicamente los intercambios en el forum de grupos separados entre alumnos y con la profesora y los textos adjuntos a dichos intercambios. Como información complementaria, se recogió el plan docente de la asignatura y los diferentes materiales elaborados por la profesora, tanto si el acceso a los mismos se dio a través de la plataforma Moodle como si fueron facilitados en la actividad presencial.

Para cumplir con el objetivo previsto de identificar y caracterizar las ayudas educativas se elaboró - a partir de los datos y en contraste continuo con la perspectiva teórica de partida - un protocolo de análisis. Dicho protocolo consta de tres dimensiones, de las subdimensiones correspondientes a cada dimensión, y de las categorías de cada subdimensión (ver Tabla 1).

Tabla 1. Dimensiones, subdimensiones y categorías de análisis

\begin{tabular}{|c|}
\hline Dimensión 1. Potencialidades y limitaciones de la propuesta docente \\
\hline 1.1. Potencialidades por los usos de las TIC $(P T)$ \\
\hline La participación no se rige por las exigencias de la coincidencia espacial-temporal $(P T p)$ \\
\hline Disponen de tiempo de reflexión individual para elaborar la propia contribución (PTri) \\
\hline Seguimiento de la participación por los alumnos $(P T s p)$ \\
\hline Seguimiento de las sucesivas versiones del texto según las exigencias de la tarea (PTet) \\
\hline Negociación entre alumnos de los significados sobre los contenidos (PTns) \\
\hline Seguimiento de la actividad de los alumnos para prestar ayuda educativa ( $P T s p a)$ \\
\hline Acceso a la información, a los contenidos y materiales docentes (PTaic) \\
\hline 1.2. Limitaciones por los usos de las TIC $(L T)$ \\
\hline Conocimientos y experiencias previos de participación colaborativa en el forum (LTcepf) \\
\hline Conocimientos y experiencias previos de la tarea de escritura colaborativa online (LTceto) \\
\hline $\begin{array}{l}\text { Conocimientos y experiencias previas del debate online de los significados sobre los conte- } \\
\text { nidos (LTcedo) }\end{array}$ \\
\hline $\begin{array}{l}\text { Conocimientos y experiencia de la profesora en orientar la tarea de escritura colaborativa } \\
\text { online (LTcept) }\end{array}$ \\
\hline Comunicación entre alumnos para gestionar la escritura conjunta del texto (LTcgec) \\
\hline Comunicación entre alumnos para dar estructura al texto (LTcet) \\
\hline Comunicación entre alumnos para debatir los significados sobre el contenido. (LTcds) \\
\hline Comunicación entre profesora y alumnos para recibir ayudas a sus necesidades (LTcpa) \\
\hline 1.3. Potencialidades por la presencialidad $(P P)$ \\
\hline Ajustar las ayudas educativas al aprendizaje de los contenidos (PPaac) \\
\hline Ajustar las ayudas al aprendizaje de la participación colaborativa (PPaaco) \\
\hline Ajustar las ayudas a la realización de la tarea de escritura colaborativa ( $P P a a$ \\
\hline
\end{tabular}


Mejorar el aprendizaje de contenidos (PPmac)

Mejorar el rendimiento académico del grupo (PPmra)

1.4. Limitaciones por la presencialidad $(L P)$

Ayudas a la tarea de escritura colaborativa (LPat)

Dimensión 2. Ayudas educativas

\subsection{Ayudas a la gestión de la participación $(G P)$}

Representarse qué es la colaboración. (GPrc)

Normas de colaboración. (GPnc)

Continuidad de la colaboración (GPcc)

Valoración del cumplimento de las normas de colaboración (GPvcn)

Acuerdos sobre normas de colaboración diferentes a las planteadas por la profesora (GPand)

\subsection{Ayudas a la gestión de la tarea $(G T)$}

Representación del texto como producto final (GTrpf)

Representación del proceso para llevar a cabo la tarea (GTrpt)

Normas de elaboración del texto: aspectos formales (GTnaf)

Normas de estructura del texto (GTnet)

Normas del proceso de escritura colaborativa (GTnpe)

Seguimiento y resolución de dudas sobre normas de los aspectos formales del texto (GTsnf)

Seguimiento y resolución de dudas sobre las normas del proceso de la tarea (GTsnpt)

Valoración del proceso de tarea seguido (GTvpt)

Valoración de los resultados o producto final (GTvpf)

2.3. Ayudas a la gestión de la elaboración de significado sobre el contenido (GS)

Normas de selección del contenido del texto (GSnsc)

Normas de debate y negociación conjunta de significados (GSnds)

Seguimiento y resolución de dudas sobre el contenido del texto (GSsdc)

Seguimiento y supervisión de la elaboración de significados compartidos (GSsesc)

Valoración del nivel de significado alcanzado en el producto final (GSvns)

Valoración grado en que persisten dudas (GSvpd)

Compartir los criterios de evaluación del aprendizaje (GScce)

\section{Dimensión 3. Resultados de la propuesta docente}

\subsection{Resultados del seguimiento $y$ ayudas de la profesora $(R)$}

Seguimiento de la elaboración del texto en las tutorías presenciales $(R s t p)$

Acceso continuado online a la información, los contenidos de aprendizaje y materiales docentes (Racm)

Seguimiento online de la elaboración del texto (Rso)

Concreción de las normas de participación de los alumnos en las tutorías presenciales

(Rcntp)

Comunicación e inmediatez del feedback online con la profesora (Rcifo)

Condiciones del seguimiento continuado por el profesor (Rcscp)

Estructura de participación global combinando ayudas presenciales y online (Repg)

\subsection{Resultados de aprendizaje $(R A)$}

Aprendizaje de los contenidos $(R A c)$

Conocimiento del proceso de escritura colaborativo (RApec)

Aprendizaje de la colaboración ( $R A c o)$

Uso adecuado de la tecnología del forum para el aprendizaje (RAut) 


\subsection{Resultados de la gestión de los grupos $(R G G)$}

Gestión de la participación colaborativa online $(R G G p)$

Gestión de la tarea $(R G G t)$

Gestión del debate online sobre los significados de los contenidos (RGGs)

\section{Análisis de los datos}

La metodología utilizada para el análisis de la actividad de elaboración colaborativa de textos escritos es la metodología cualitativa (Strauss y Corbin, 2002, Grounded Theory) y el análisis de casos (Yin, 2006).

En una primera fase, tres equipos independientes, compuestos cada uno por dos investigadores, identificaron los elementos que hipotéticamente respondían a potenciales categorías de cada dimensión y subdimensión de la Tabla 1 y contrastaron entre sí sus interpretaciones para elaborar y afinar progresivamente una definición empírica y teórica compartida de cada una de ellas. También se analizaron las entrevistas iniciales y finales y los diarios de las profesoras, separadamente de las entrevistas a los alumnos. En concreto se identificaron: las potencialidades y limitaciones de la propuesta docente para el aprendizaje; las expectativas sobre las ayudas educativas y las realmente prestadas, y el grado de cumplimiento de las expectativas iniciales. El resultado del análisis se contrastó y completó con los datos electrónicos y audiovisuales recogidos.

En una segunda fase, una vez establecido el protocolo definitivo de análisis, éste fue aplicado a los datos. Para ello se siguió un procedimiento iterativo de consenso entre codificadores expertos independientes que asignaban las categorías a los datos y contrastaban los resultados. Las categorías se contrastaron empíricamente y también se siguió perfeccionando su caracterización y operativización correspondiente.

Posteriormente, en una tercera fase, considerando los resultados de la aplicación del protocolo, se procedió a la triangulación de los datos. El proceso seguido en cada caso fue el siguiente: a) se identificó por separado la percepción de las Potencialidades y limitaciones de la propuesta docente de la profesora y la percepción de los alumnos (Dimensión 1); b) se identificaron las Ayudas educativas a partir de los datos procedentes de fuentes de informa- 
ción relativas tanto a profesores como a alumnos (Dimensión 2); y finalmente, se establecieron los Resultados de la propuesta docente (Dimensión 3). En conjunto, el proceso de interpretación siguió los principios de la Grounded Theory y sirvió para configurar los resultados del análisis que se exponen a continuación.

\section{Resultados}

A continuación nos ocuparemos de los resultados del Caso 1 (C1) y del Caso 2 (C2). Para evitar repeticiones innecesarias, las tablas que acompañan la presentación del C1 aportan los datos de ambos casos que, posteriormente, se comentan por separado.

\section{Resultados del Caso 1}

En cuanto a las características generales de la propuesta, el $\mathrm{C} 1$ presenta un diseño que combina la presencialidad y la virtualidad a lo largo de la SD, variando la prioridad de una y otra en la configuración la actividad conjunta. Se identificaron tres períodos de duración y prioridad diferentes. En el período inicial (dos semanas) la participación de los alumnos es presencial, combinada con la del aula virtual para acceder a la información, a los materiales docentes y a la formación de grupos de trabajo online. En el período intermedio (cuatro semanas) la tarea se desarrolla online con una tutoría presencial. En el período final (una semana), la participación de los alumnos se da en sesiones presenciales de carácter general y de tutoría de pequeño grupo. La estructura de participación de los alumnos en la SD a nivel global es muy pautada, aunque su actividad en torno a la tarea es abierta, basada fundamentalmente en recomendaciones y orientaciones de carácter general. La gestión por los alumnos de un proceso de estas características les exige altos niveles de interacción sostenida y recíproca, entre ellos y con la profesora (para más información al respecto, consultar Mauri, Colomina y Ginesta, 2011).

En relación a las potencialidades y limitaciones de la propuesta docente, la profesora y los alumnos diferencian entre lo que les resulta característico de la participación virtual y de la participación presencial (Tablas 2 y 3). 
Tabla 2. Posibilidades de la propuesta docente

\begin{tabular}{|c|c|c|c|c|c|c|c|c|c|c|c|c|}
\hline & \multicolumn{7}{|c|}{ Posibilidades por usos de las TIC } & \multicolumn{5}{|c|}{$\begin{array}{l}\text { Posibilidades por la presen- } \\
\text { cialidad }\end{array}$} \\
\hline & $\begin{array}{l}P T \\
p\end{array}$ & $\begin{array}{l}P T \\
r i\end{array}$ & $\begin{array}{l}P T \\
s p\end{array}$ & $\begin{array}{l}P T \\
\text { et }\end{array}$ & $\begin{array}{l}P T \\
n s\end{array}$ & $\begin{array}{l}P T \\
\text { spa }\end{array}$ & $\begin{array}{l}P T \\
\text { aic }\end{array}$ & $\begin{array}{l}P P \\
\text { aac }\end{array}$ & $\begin{array}{l}P P \\
\text { aat }\end{array}$ & $\begin{array}{l}P P \\
\text { aaco }\end{array}$ & $\begin{array}{l}P P \\
m a c\end{array}$ & $\begin{array}{l}P P \\
m r a\end{array}$ \\
\hline C1P & & & & $\mathrm{x}$ & & $\mathrm{x}$ & $\mathrm{X}$ & & & & $\mathrm{x}$ & \\
\hline C1A & & $\mathrm{X}$ & $\mathrm{X}$ & $\mathrm{x}$ & $\mathrm{x}$ & $\mathrm{x}$ & $\mathrm{x}$ & $\mathrm{x}$ & $\mathrm{X}$ & $\mathrm{x}$ & $\mathrm{x}$ & $\mathrm{x}$ \\
\hline $\mathbf{C 2 P}$ & & & $\mathrm{x}$ & & $\mathrm{x}$ & $\mathrm{x}$ & & $\mathrm{X}$ & & & $\mathrm{x}$ & $\mathrm{x}$ \\
\hline $\mathbf{C 2 A}$ & & $\mathrm{X}$ & $\mathrm{X}$ & $\mathrm{X}$ & $\mathrm{X}$ & $\mathrm{X}$ & $X$ & $\mathrm{X}$ & & $\mathrm{X}$ & $\mathrm{X}$ & $\mathrm{X}$ \\
\hline
\end{tabular}

Con respecto a los usos de las TIC, la profesora afirma que permiten a los alumnos el acceso a las sucesivas versiones del texto y el seguimiento e implicación continuados en su elaboración, y a ella misma también le permiten realizar el seguimiento del desempeño de la tarea para prestar ayuda educativa. Entiende que la mejora del aprendizaje de los contenidos depende de ambos procesos. Al mismo tiempo, la profesora plantea que dichos usos limitan la comunicación entre alumnos para una adecuada gestión de la tarea y para un debate efectivo de los significados sobre los contenidos. Afirma que la falta de experiencia en el debate de significados con TIC y en tareas de escritura colaborativa asíncrona -tanto por parte de los estudiantes para realizarlo, como de la profesora para apoyarles- limita las potencialidades de la propuesta docente. Asimismo, la profesora afirma que las situaciones cara a cara (sesiones de explicación de los contenidos; tutorías finales), mejoran el aprendizaje de los contenidos, mediante la asistencia más ajustada a las necesidades de comprensión de los estudiantes.

Tabla 3. Limitaciones de la propuesta docente

\begin{tabular}{|c|c|c|c|c|c|c|c|c|c|}
\hline & \multicolumn{8}{|c|}{ Limitaciones por usos de las TIC } & \multirow{3}{*}{\begin{tabular}{|l|}
$\begin{array}{l}\text { Limitaciones por la } \\
\text { oresencialidad }\end{array}$ \\
\\
$L P$ \\
$a t$
\end{tabular}} \\
\hline & \multicolumn{4}{|c|}{$\begin{array}{l}\text { Conocimiento y experiencia } \\
\text { previos }\end{array}$} & \multicolumn{4}{|c|}{ Comunicación } & \\
\hline & $\begin{array}{l}\text { LT } \\
\text { cepf }\end{array}$ & $\begin{array}{l}\text { LT } \\
\text { ceto }\end{array}$ & $\begin{array}{l}\text { LT } \\
\text { cedo }\end{array}$ & $\begin{array}{l}\text { LT } \\
\text { cept }\end{array}$ & $\begin{array}{l}L T \\
\text { cgec }\end{array}$ & $\begin{array}{l}L T \\
\text { cet }\end{array}$ & $\begin{array}{l}L T \\
c d s\end{array}$ & $\begin{array}{l}L T \\
c p a\end{array}$ & \\
\hline C1P & $\mathrm{x}$ & $\mathrm{x}$ & $\mathrm{x}$ & $\mathrm{x}$ & $\mathrm{x}$ & & $\mathrm{x}$ & & \\
\hline$\overline{\mathrm{C1A}}$ & $\mathrm{X}$ & $\mathrm{X}$ & & $\mathrm{X}$ & $\mathrm{x}$ & $\mathrm{X}$ & $\mathrm{x}$ & $\mathrm{x}$ & $\mathrm{x}$ \\
\hline $\mathbf{C 2 P}$ & $\mathrm{x}$ & $\mathrm{x}$ & & $\mathrm{X}$ & $\mathrm{x}$ & & $\mathrm{x}$ & $\mathrm{x}$ & \\
\hline$\overline{\mathrm{C2A}}$ & & & $\mathrm{x}$ & $\mathrm{x}$ & $\mathrm{x}$ & & $\mathrm{x}$ & $\mathrm{x}$ & \\
\hline
\end{tabular}

Los alumnos coinciden con la profesora en las potencialidades de la propuesta docente por el uso de las TIC y añaden otras derivadas del carácter escrito de la comunicación: poder reflexionar individualmente sobre la contribución de otros para elaborar la propia; gestionar la 
colaboración en la tarea, y negociar el significado de los contenidos de aprendizaje. Aunque coinciden con la docente en casi todas las limitaciones de la propuesta, apuntan otras dos nuevas: la dificultad de la comunicación online para estructurar conjuntamente el texto y para comunicarse con la profesora. Los alumnos consideran que las sesiones presenciales posibilitan un mayor ajuste de las ayudas al aprendizaje de los contenidos y la colaboración entre iguales. Entre las limitaciones de las sesiones de presentación de la SD indican que la pautación de los pasos a seguir en la tarea de escritura colaborativa fue insuficiente y que hubieran necesitado más ayuda sobre cómo hacer progresar conjuntamente un texto. A diferencia de la profesora, que atribuye ciertas limitaciones a las condiciones institucionales (tiempo, número de alumnos, etc.), los alumnos señalan la necesidad de una mayor pautación de la actividad durante la tarea, presencial y virtual, y mayor rapidez y contingencia en las respuestas a sus demandas de ayuda virtuales.

Las ayudas educativas identificadas por profesora y alumnos se refieren tanto a la gestión de la participación, como a la gestión de la tarea y a la gestión del significado. En cuanto a la gestión de la participación (Tabla 4), al inicio del proceso de enseñanza y aprendizaje se dieron ayudas educativas para representarse y compartir en qué consiste una participación colaborativa online (por ejemplo, las ideas de los alumnos sobre la colaboración fueron debatidas en clase). También tuvieron ayudas para establecer normas de participación (por ejemplo, normas relativas a usar colores para identificar a cada miembro en cada contribución; establecer turnos; definir roles; etc.). Asimismo se impulsó la creación de normas de colaboración por acuerdo. A lo largo de dicho proceso se abordaron dudas sobre las normas de colaboración, y se decidieron otras nuevas. En las tutorías presenciales al final del bloque se valoró el cumplimiento y la eficacia de las normas de participación colaborativa.

Tabla 4. Ayudas educativas a la gestión de la participación

\begin{tabular}{|l|l|l|l|l|l|}
\hline & GPrc & GPnc & GPcc & GPvcn & GPand \\
\hline G1 & $\mathrm{x}$ & $\mathrm{x}$ & $\mathrm{x}$ & $\mathrm{x}$ & $\mathrm{x}$ \\
\hline G2 & & $\mathrm{x}$ & $\mathrm{x}$ & $\mathrm{x}$ & \\
\hline
\end{tabular}

En cuanto a la gestión de la tarea (Tabla 5), se dieron ayudas para representarse la tarea; en concreto, el texto como producto (por ejemplo, se presentaron modelos de textos explicativos que mostraran explícitamente la estructura de textos de este tipo). 
Tabla 5. Ayudas educativas a la gestión de la tarea

\begin{tabular}{|l|l|l|l|l|l|l|l|l|l|}
\hline & \multicolumn{3}{|l|}{ Representación } & \multicolumn{3}{l|}{ Normas } & \multicolumn{3}{l|}{ Seguimiento/Valoración } \\
\cline { 2 - 10 } & GTrpf & GTrpt & GTnaf & GTnet & GTnpe & GTsnf & GTsnpt & GTvpt & GTvpf \\
\hline G1 & $\mathrm{x}$ & $\mathrm{x}$ & & $\mathrm{x}$ & $\mathrm{x}$ & $\mathrm{x}$ & & $\mathrm{x}$ & $\mathrm{x}$ \\
\hline G2 & $\mathrm{x}$ & $\mathrm{x}$ & & $\mathrm{x}$ & $\mathrm{x}$ & $\mathrm{x}$ & $\mathrm{x}$ & & $\mathrm{x}$ \\
\hline
\end{tabular}

Asimismo, los alumnos recibieron ayudas para representarse un modelo de realización de la tarea (partir de un borrador inicial compartido; realizar contribuciones de mejora, ampliación y profundización; realizar revisiones puntales; elaborar síntesis antes de seguir progresando, etc.). Se les ayudó al inicio para que establecieran normas de estructuración del texto y de escritura colaborativa. A lo largo del proceso, recibieron ayudas de la profesora en la resolución de dudas sobre las normas de escritura colaborativa y sobre las relativas a los aspectos formales y estructurales del texto. Una vez finalizado el proceso, las ayudas al aprendizaje se dieron mediante la valoración del proceso seguido en la tarea y del producto final en las tutorías presenciales.

En cuanto a la gestión del significado (Tabla 6), profesora y alumnos coinciden en que existieron ayudas para identificar y seleccionar el contenido y evitar lagunas en la selección del mismo.

Tabla 6. Ayudas educativas a la gestión del significado de los contenidos

\begin{tabular}{|l|l|l|l|l|l|l|l|}
\hline \multirow{2}{*}{} & \multicolumn{2}{|l|}{ Normas } & \multicolumn{3}{|l|}{ Seguimiento/Valoración } & Criterios \\
\cline { 2 - 8 } & GSnsc & GSnds & GSsdc & GSsesc & GSvns & GSvgd & GScce \\
\hline G1 & $\mathrm{x}$ & & $\mathrm{x}$ & & $\mathrm{x}$ & & $\mathrm{x}$ \\
\hline G2 & $\mathrm{x}$ & & $\mathrm{x}$ & & $\mathrm{x}$ & $\mathrm{x}$ & \\
\hline
\end{tabular}

La ayuda a la elaboración, centrada en la confección de un índice, se proporcionó a los alumnos en una sesión presencial, después de que la profesora constatara que las dificultades de elaboración seguían manteniéndose a pesar de los esfuerzos por resolverlas. También se reciben ayudas para resolver dudas sobre el contenido que debería incluirse en el texto (qué contenido; qué grado de complejidad, etc.). Finalizado el bloque, en las tutorías presenciales y una vez calificados los textos por la profesora, se recibieron ayudas para valorar el grado de aprendizaje mostrado en el producto final y solucionar las dudas que persistían. 
$\mathrm{Al}$ referirse a los resultados alcanzados profesora y alumnos coinciden en que la propuesta docente mejora los tres aspectos siguientes: una más adecuada oferta de ayudas; unos mejores resultados de aprendizaje, y una mejor gestión de la tarea por parte de los grupos. Aún así, señalan que el seguimiento y la pautación de la elaboración del texto online por parte de la profesora necesitarían mejorar respondiendo a las demandas de los estudiantes con inmediatez. Y profesora y alumnos indican que deberían también mejorar las condiciones de enseñanza y aprendizaje (muchos alumnos y grupos de alumnos de primer curso con una única profesora) y los conocimientos y las experiencias iniciales de ambos (profesora y alumnos) sobre la tarea de escritura colaborativa con TIC (Tabla 7).

Tabla 7. Resultados del seguimiento de la actividad y de las ayudas de la profesora

\begin{tabular}{|l|l|l|l|l|l|l|l|}
\hline & Rstp & Racm & Rso & Rcntp & Rcifo & Rcscp & Repg \\
\hline G1 & $\mathrm{x}$ & $\mathrm{x}$ & $\mathrm{x}$ & $\mathrm{x}$ & & & $\mathrm{x}$ \\
\hline G2 & $\mathrm{x}$ & $\mathrm{x}$ & $\mathrm{x}$ & $\mathrm{x}$ & $\mathrm{x}$ & $\mathrm{x}$ & \\
\hline
\end{tabular}

Por último, los participantes afirman que la propuesta docente (Tabla 8) consigue que los alumnos alcancen resultados positivos de aprendizaje de los contenidos y del proceso de escritura colaborativa con TIC para aprender. Sin embargo, se siguen detectando dificultades en los procesos de gestión online de la participación, de la tarea y de los significados.

Tabla 8. Resultados de aprendizaje y de la gestión de los grupos

\begin{tabular}{|l|l|l|l|l|l|l|l|}
\hline & \multicolumn{4}{|l|}{ Resultados de aprendizaje } & \multicolumn{3}{l|}{ Resultados gestión grupos } \\
\cline { 2 - 8 } & $R A c$ & RApec & RAco & $R A u t$ & $R G G p$ & $R G G t$ & $R G G s$ \\
\hline G1 & $\mathrm{x}$ & $\mathrm{x}$ & $\mathrm{x}$ & $\mathrm{x}$ & $\mathrm{x}$ & & $\mathrm{x}$ \\
\hline G2 & $\mathrm{x}$ & & $\mathrm{x}$ & $\mathrm{x}$ & & & \\
\hline
\end{tabular}

\section{Interpretación del Caso 1}

La utilización de un entorno virtual y de una herramienta que no ha sido creada específicamente para apoyar tareas de escritura colaborativa online para el aprendizaje de contenidos, llevó a la profesora a un alto grado de formalización de la propuesta tecnopedagógica de la SD, predefiniendo una serie de condiciones que promovieran una estructura de participación que consideraba beneficiosa para el aprendizaje de los alumnos. El diseño de la propuesta docente pretendía compensar tanto las limitaciones de la colaboración mediada por el fo- 
rum, como las propias de los conocimientos iniciales de los alumnos ayudándoles a representarse las exigencias de la tarea y a definir normas reguladoras de los procesos de interacción.

La ayuda educativa de la profesora del $\mathrm{C} 1$ se presenta a niveles distintos y en momentos diferentes de la actividad conjunta. En un primer nivel encontramos ayudas ligadas al planteamiento de la actividad a nivel global que se dirigen a todos los alumnos y a presentar y dar sentido a la tarea, a planificar la actividad y a representarse los resultados de aprendizaje. En un segundo nivel encontramos ayudas directas a los grupos que remiten a aspectos específicos de realización de la tarea (ayudas a representarse las condiciones y exigencias de la tarea, las características de los productos a entregar; el proceso global a seguir para elaborar el producto en las condiciones establecidas, etc.). La ayuda de la profesora se sitúa en momentos diferentes del flujo de la actividad conjunta (ayuda al inicio de la actividad; ayuda durante el proceso; ayuda a posteriori) e implica formas distintas de llevar a cabo dicha influencia educativa. En el C1, las ayudas al inicio se asocian a una modalidad de interacción presencial y virtual; los dispositivos de ayuda durante el proceso se asocian a la interacción virtual -y puntualmente presencial-, y las ayudas a posteriori se asocian de nuevo a la interacción presencial.

En cuanto a los aspectos de la actividad conjunta sobre los cuales se ofrece la ayuda, es posible concluir a partir de los resultados, que al inicio aparecen ayudas a la gestión de la participación, a la gestión de la tarea y a la elaboración del significado. Son ayudas a la participación colaborativa (normas de implicación colaborativa), a la representación del tipo de texto (directrices proporcionadas sobre su estructura), al contenido del texto, a la selección de los contenidos sobre los que deben elaborarse los significados, (acceso a las lecturas; explicaciones de la profesora, síntesis), a la representación y elaboración del proceso a seguir en la tarea (modelos y directrices proporcionadas por el profesor sobre la escritura colaborativa), y al debate sobre el significado (normas sobre como debatir los significados que otros aportan sobre los contenidos). Sin embargo, durante el proceso, las ayudas se centran fundamentalmente en seleccionar, hacer avanzar el contenido del texto y mejorar su estructura, y en las posibilidades de colaboración entre iguales. Así, las ayudas se concentran al inicio del proceso y las que se dan durante el mismo se centran en el producto y no en el proceso de gestión de la actividad conjunta por parte de los alumnos. En este sentido, existe una diferencia fundamental entre ayudarles a representarse dimensiones y aspectos diferentes de la actividad 
conjunta y ayudarlos a llevarla a cabo convenientemente, gestionando la colaboración de manera autónoma.

\section{Resultados del Caso 2}

En cuanto a las características generales de la propuesta, el Caso 2 combina también las posibilidades que ofrece la virtualidad con las propias de la presencialidad. Pero, a diferencia del C1, la presencialidad es la que sostiene, muy por encima de lo que lo hace la virtualidad, la interacción de profesora y alumnos a lo largo del proceso de enseñanza y aprendizaje. Existen dos períodos diferentes (inicial e intermedio-final) de organización de la estructura de participación. En el período inicial (dos semanas de duración) los grupos de alumnos acceden a la información, los contenidos y materiales docentes en el aula ordinaria, y a los materiales del aula virtual. Dicho período finaliza con el envío online del índice del texto a la profesora. En el período intermedio-final (cinco semanas de duración) se llevan a cabo tutorías semanales presenciales de seguimiento de la profesora en relación con la tarea desarrollada por los grupos. La profesora usa las subtareas de elaboración del texto -planificación o índice (seleccionar el contenido); elaboración o ejecución de la planificación (completar sus partes y revisar progresivamente el texto) y evaluación-revisión (cierre)- para estructurar la participación presencial entre alumnos y con la profesora. Sin embargo, deja más abierto lo que pueden hacer los alumnos y la profesora online.

Para comentar como la profesora y los alumnos se refieren a las posibilidades y limitaciones de la propuesta docente del $\mathrm{C} 2$ es necesario remitirse de nuevo a los datos que constan en las Tablas 2 y 3. La profesora del C2 coincide con la del C1 respecto a las posibilidades de la propuesta docente y añade además que el uso de las TIC ofrece opciones para el seguimiento de la participación de todos los implicados en la actividad. Desde su punto de vista, no sólo es útil a los alumnos para gestionar el proceso, sino también a la profesora para identificar la contribución a la tarea de cada estudiante. En cuanto a las limitaciones de la propuesta docente, considera también que los usos de las TIC limitan la comunicación entre alumnos y con la profesora. Identifica como limitaciones también la falta de conocimiento y experiencia de los estudiantes en la participación en el foro, y la de estudiantes y profesora sobre tareas de escritura colaborativa asíncrona. En cuanto a lo que aportan a la propuesta docente las situaciones de interacción cara a cara, la profesora afirma que permiten ajustar y diversificar la ayuda educativa a las necesidades de aprendizaje y que contribuyen a mejorarlo, así como también el 
rendimiento académico de los estudiantes. A su juicio, las limitaciones de la propuesta docente no provienen en ningún caso de la inclusión de situaciones presenciales.

Los alumnos del C2 coinciden con su profesora en las potencialidades del uso de las TIC y añaden otras como: reflexionar individualmente sobre la contribución de otros para elaborar la propia; gestionar la participación colaborativa en la tarea de los miembros del grupo, negociar el significado de los contenidos de aprendizaje, y posibilitar el acceso continuado a la información, los contenidos y materiales docentes. Coinciden con la docente respecto a las limitaciones de la propuesta debidas al uso de las TIC pero, a diferencia de ésta, señalan que su falta de conocimiento y experiencia en el debate de los significados sobre los contenidos online también las incrementa. Asimismo los alumnos coinciden con su profesora en las posibilidades educativas de la propuesta gracias a las situaciones de interacción cara a cara y añaden que les permiten gestionar mejor la tarea.

Las ayudas educativas identificadas por la profesora y alumnos se refieren a la gestión de la participación, de la tarea y del significado. En cuanto a la gestión de la participación colaborativa (Tabla 4), al inicio de la SD se dieron ayudas educativas para establecer normas de participación y, a lo largo de la misma, se abordaron dudas sobre su uso y se valoró su cumplimiento. En cuanto a la gestión de la tarea (Tabla 5), se dieron ayudas para representarse la tarea e identificar el proceso de escritura colaborativa con los tres momentos de elaboración del texto ya señalados. También se les ayudó a establecer normas de colaboración y de estructuración del texto, y en la resolución de dudas sobre los aspectos formales y estructurales del texto. Además, antes de entregar el producto final, recibieron ayudas presenciales para valorar la tarea y el texto como producto. En cuanto a la gestión del significado (Tabla 6), profesora y alumnos coinciden en que existieron ayudas en forma de normas para identificar y seleccionar el contenido del texto y en que, a lo largo del proceso, las recibieron para resolver dudas sobre los significados del contenido de aprendizaje, para valorar el grado en que persistían las dudas sobre los significados y el aprendizaje alcanzado. Las ayudas se prestaron en las tutorías presenciales. No se señalan ayudas a la elaboración conjunta de esos significados ya que la profesora se centra en seguir los productos parciales y el producto final.

Respecto a los resultados alcanzados, profesora y alumnos coinciden en que la propuesta mejora los tres aspectos siguientes: una más adecuada oferta de ayudas; unos mejores resultados de aprendizaje, y una mejor gestión de la tarea por parte de los grupos. Respecto a la co- 
municación entre profesora y alumnos, los resultados muestran que necesitaría mejorar el seguimiento y la pautación de la elaboración del texto online por parte de la profesora. Existieron limitaciones en la comunicación entre alumnos y con la profesora online que conllevaron que las TIC se utilizaran, esencialmente, para intercambiar los productos que se iban elaborando. A esas limitaciones cabe sumar también la falta de conocimiento y experiencia de profesora y alumnos sobre el desarrollo online de la tarea colaborativa (Tabla 7), y el hecho de que las ayudas estuvieron fundamentalmente centradas en el texto y no tanto en la gestión de la actividad conjunta. En relación a los resultados de aprendizaje (Tabla 8), profesora y alumnos coinciden en señalar que la participación en la propuesta docente consigue que alcancen resultados positivos en el aprendizaje de los contenidos y la colaboración, pero no en el uso de la virtualidad para aprender. Esto último se ve apoyado también con los siguientes resultados: los grupos han detectado dificultades en la gestión online de su participación colaborativa, en la gestión de la tarea online, y en el debate online sobre los significados de los contenidos durante el proceso. Los alumnos afirman que también existieron limitaciones en su gestión de la participación en las tutorías presenciales.

\section{Interpretación del Caso 2}

La profesora consiguió organizar, implementar y andamiar los procesos propios de la escritura colaborativa para que los alumnos lograran ser autónomos en construir conocimiento, en la colaboración y el aprendizaje de los contenidos. Para ello, igual que ocurre en el C1, dotó de un alto grado de formalización a la propuesta docente global predefiniendo las condiciones para que los alumnos se implicaran en determinadas formas de interacción que consideraba beneficiosas para su aprendizaje. En este caso, dio un gran peso a las sesiones presenciales y definió la estructura de participación de los alumnos en la tarea en torno a las fases de elaboración del texto. También vinculó el seguimiento de los resultados a las tutorías presenciales en las que ejercía directamente el control sobre los productos parciales de la tarea. Las ayudas se centraron en controlar directamente el producto. En lo relativo al texto las ayudas de la profesora se dirigieron fundamentalmente a los aspectos formales, estructurales y en garantizar la identificación de los contenidos y su comprensión significativa. La profesora consideró el espacio on-line como un repositorio de documentos, y siguió la presencia -o node los alumnos online, pero no valoró la calidad de sus contribuciones. 
Fundamentalmente se identifican ayudas al inicio y durante el proceso. Las ayudas al inicio se dan en situación presencial y virtual; las ayudas durante el proceso se dan en situación presencial y, muy puntualmente, virtual. Al inicio de la actividad aparecen ayudas a: el tipo de texto (directrices proporcionadas sobre aspectos formales y de estructura); a la comprensión de los contenidos teóricos del bloque y, puntualmente, a la participación colaborativa (directrices de implicación colaborativa). Sin embargo, durante el proceso las ayudas se centran en el producto, en seleccionar y hacer avanzar el contenido del texto, y en mejorar sus aspectos formales y de estructura. En definitiva, la profesora proporciona ayudas variadas, en diferentes momentos y sobre diferentes aspectos de la tarea, pero regula escasamente los procesos comunicativos entre estudiantes para la gestión autónoma de la tarea y para la negociación de los significados.

\section{Discusión y Conclusiones}

Al inicio de este escrito afirmábamos que el nuevo marco de desarrollo de la docencia con TIC modificaba las condiciones de la construcción del conocimiento entre alumnos en la tarea de escritura colaborativa en el espacio forum del Moodle y que, por lo tanto, era necesario profundizar en su naturaleza y características. Se trataba de conocer qué aspectos de la construcción del conocimiento resultaban favorecidos por la actuación del docente.

Los resultados muestran un elevado grado de esfuerzo por parte del profesorado para organizar una propuesta docente capaz de sostener la construcción colaborativa del conocimiento basada en la escritura de textos con Moodle. Dicho esfuerzo se debe, en parte, a que el forum es una herramienta tecnológica que no está especialmente diseñada para potenciar el aprendizaje en este tipo de tareas y, en consecuencia, los docentes se encuentran con la necesidad de elaborar una propuesta -su diseño e implementación- que contribuya al máximo a hacer posible el aprendizaje en dichas condiciones. Pero también se debe a que, desde el inicio, el profesorado se muestra altamente consciente de las dificultades de los alumnos para trabajar colaborativamente de manera virtual, de su desconocimiento de las características estructurales y funcionales de los textos académicos, y de las dificultades del proceso de composición escrita en colaboración en el forum.

En respuesta a esta situación el profesorado de la muestra desarrolla una tarea docente ingente proporcionando ayudas educativas diversas a los alumnos que se sitúan en diferentes 
niveles (Mauri, Colomina y Ginesta, 2011). En el nivel del diseño, la planificación de la propuesta docente muestra un alto grado de concreción. En el nivel del desarrollo de la propuesta docente, los resultados de la investigación han puesto de relieve que las ayudas educativas se han proporcionando sobre diferentes aspectos de la actividad, utilizando recursos diversos, y que se han realizado en distintos momentos del proceso. Las ayudas a la actividad de los alumnos al inicio, durante y al final de la tarea se han distribuido de modo desigual entre la participación, la gestión de la tarea misma y la elaboración de significados, y también se han ejecutado de forma diversa. Así, los resultados muestran una gran cantidad y variedad de ayudas al inicio de la actividad centradas en facilitar que los alumnos: (i) elaboren una representación conjunta de las exigencias y normas de participación, potencialmente favorecedora de su gestión; (ii) ayudas a la gestión de la tarea misma (por ejemplo, ayudas a elaborar una representación compartida de la tarea; a planificar el texto de manera conjunta, a decidir conjuntamente la información a incluir en el texto y el orden en que deberá presentarse; a concretar los aspectos formales y las características del producto final, etc.); y ayudas a elaborar el contenido de manera significativa. Pero, mientras las ayudas al inicio de la tarea han sido muchas y muy variadas, las ayudas durante la tarea se han centrado mayoritariamente en la gestión para hacerla progresar decidiendo los contenidos y mejorando los aspectos estructurales y formales del texto como producto final esperado. Además, las ayudas se han dado sobre las sucesivas versiones del texto, es decir, no sobre la actividad de los alumnos para escribir colaborativamente para aprender, sino sobre los resultados de esta actividad o sobre las versiones del texto. En consecuencia, no han contribuido a hacer avanzar la gestión autónoma de los estudiantes del proceso de construcción conjunta del conocimiento soportada en la escritura, ni tampoco a la elaboración conjunta del significado. Hubiera sido necesario centrar las ayudas no tanto en validar los resultados de la actividad en la versión " $n$ " del texto sino en consolidar una estrategia de construcción conjunta del conocimiento mediante la escritura: (i) diseñando una estructura de la tarea realmente colaborativa, no acumulativa; (ii) favoreciendo la escritura estratégica, con ayudas a la planificación del texto (por ejemplo, favoreciendo el desarrollo y revisión del mismo por parte de los alumnos, evitando substituirles en ambos procesos; ofreciéndoles instrumentos y apoyos para llevarlos a cabo; ayudándoles a hacer explícitos los problemas que encuentran y a solucionarlos de manera conjunta); (iii) favoreciendo que los estudiantes interactúen entre ellos y con el conocimiento mientras escriben, haciendo progresar la elaboración del significado (por ejemplo, propiciando que las contribuciones al texto de cada estudiante expresen, a la vez, tanto el grado en que comparten dicho texto, como argumentando propuestas de modificación y cambio). 
En consecuencia, cabe recomendar que el profesor medie en el proceso de interacción entre alumnos y no únicamente en el control del resultado. Esta mediación, acorde con los principios constructivistas, sin duda pasaría por establecer una mejor y más adecuada coordinación de las ayudas prestadas a nivel presencial y virtual, y una mayor diversidad y grado de ajuste de las ayudas a las necesidades de los alumnos especialmente durante el proceso. Tal como ya han puesto de relieve otros autores (Dillenbourg, 2002; Onrubia y Engel, 2009; Shuters, 2010; Stahl, Koschmann y Suthers, 2006; Weinberger, Ertl, Fischer y Mandl, 2005), el uso de scripts para promover la construcción del conocimiento podría resultar de utilidad para centrar la actividad en los elementos fundamentales de la construcción conjunta del conocimiento, favoreciendo el control y la toma de decisiones compartidas sobre las diferentes dimensiones de la actividad colaborativa de tareas de escritura colaborativa para aprender. Finalmente, el estudio confirma la importancia de promover un uso de las TIC centrado en sostener la gestión de la interactividad para el aprendizaje de los estudiantes en este tipo de tareas (Rouke y Kanuka, 2007; Stahl, 2005; Veldhuis-Diermanse, 2002; Van Drie, Van Boxtel, Jaspers y Kanselaar, 2005).

\section{Agradecimientos}

Esta investigación ha contado con la financiación obtenida en concurso público en el Programa de Recerca en Docència Universitària (REDICE-08). Proyecto A0801-16. Investigadora Principal: Teresa Mauri. Este trabajo también ha sido realizado en el marco de un proyecto de investigación financiado por la Dirección General de Investigación del Ministerio de Educación y Ciencia (EDU2009- 08891). Se puede encontrar más información en http://www.psyed.edu.es/grintie

\section{Referencias}

Castelló, M. (2009). Aprender a escribir textos académicos: ¿copistas, escribas, compiladores o escritores? En J.I. Pozo \& M. del P. Pérez Echevarría (coords.) Psicología del aprendizaje universitario: la formación en competencias (pp. 120-133). Madrid: Morata.

Castelló, M., Bañales, G., \& Vega, N. (2010).Enfoques en la investigación de la regulación de escritura académica: Estado de la cuestión. Electronic Journal of Research in Educational Psychology, 8(3), 1253-1282. 
Castelló, M., Iñesta, A., \& Monereo, C. (2009). Towards Self-regulated Academic Writing: an exploratory study with graduate students in a situated learning environment. Electronic Journal of Research in Educational Psychology, 7(3), 1107-1130.

Cerratto-Pragman, T. (2003). Collaborating with writing tools. An instrumental perspective on the problem of computer-supported collaborative activities. Interacting with Computers, $15,737-757$.

Coll, C. (2001). Lenguaje, actividad y discurso en el aula. En C. Coll, J. Palacios \& A. Marchesi (eds), Desarrollo Psicológico y educación. Psicología de la educación escolar II. (pp. 387-415). Madrid: Alianza.

Coll, C., Engel, A., \& Bustos, A. (2009). Distributed Teaching Presence and Participants'Activity Profiles: a theoretical approach to the structural analysis of Asynchronous Learning Networks. European Journal of Education, 44 (4), 521-538.

Coll, C. Mauri, T., \& Onrubia, J. (2008a). Análisis de los usos reales de las TIC en contextos educativos formales: una aproximación socio-cultural. Revista electrónica de investigación educativa, 10, (1). Consultado en http://redie.uabc.mx el 27 de septiembre de 2010.

Coll, C. Mauri, T., \& Onrubia, J. (2008b). La utilización de las tecnologías de la información y de la comunicación en la educación: del diseño tecno-pedagógico a las prácticas de uso. En C. Coll \& C. Monereo (coords). La Psicología de la educación Virtual, (pp. 74104). Madrid: Morata.

Dillenbourg, P. (2002). Over-scripting CSCL: The risks of blending collaborative learning with instructional design. En P.A. Kirschner (Ed.), Three worlds of CSCL. Can we support CSCL (pp. 61-91). Heerlen: Open Universiteit Nederland.

Lowry, P. B., Curtis, A., \& Lowry, M. R. (2004). A taxonomy of collaborative writing to improve empirical research, writing practice, and tool development. Journal of Business Communication (JBC), 41(1), 66-99.

Mauri, T., Clarà, M. \& Remesal, A. (2011). La naturaleza del discurso en la escritura colaborativa online: intersubjetividad y elaboración del significado. Infancia y Aprendizaje, 34 (2) $219-233$.

Mauri, T., Colomina, R., \& Ginesta, A. (2011). Escribir colaborativamente para aprender. Un estudio de caso centrado en las ayudas educativas del profesor en tareas con Moodle. Comunicación presentada en el VI Congreso Internacional de Psicología y Educación. Valladolid. Marzo de 2011. 
Mercer, N. (2000). Words and Minds: How We Use Language to Think Together. London: Routledge.

Onrubia, J. \& Engel, A. (2009). Strategies for collaborative writing and phases of knowledge construction in CSCL environments. Computers \& Education, 53 (4), 1256-1265.

Rourke, L. \& Kanuka, H. (2007). Barriers to online critical discourse. International Journal of Computer-Supported Collaborative Learning, 2, 105-126.

Saunders, W. (1989). Collaborative writing tasks and peer interaction. International Journal of Educational Research, 13, 101-112.

Sthal, G. (2005). Group cognition in computer assisted collaborative learning. Journal of Computer Assisted Learning, 21 (2), 79-90.

Sthal, G., Koschmann, T. \& Suthers, D. (2006). Computer-supported collaborative learning: An historical perspective. En R.K. Sawyer (Ed.) Cambridge Handbook of Learning Sciences (pp. 409-426). Cambridge: Cambridge University Press. http://GerryStahl.net/cscl/CSCL_English.pdf

Shuters, D. (2010). Roles of computacional scripts. En F. Fischer, I. Kollar \& H. Mandler (Eds.), Scripting computer-supported collaborative learning: Cognitive, Computacional and Educational Perspectives (177-189) New York: Springer.

Strauss, A \& Corbin, J. (2002). Bases de la investigación cualitativa. Técnicas y procedimien tos para desarrollar la teoría fundamentada. Bogotá. Colombia. (2a. ed.).CONTUSEditorial. Universidad de Antioquia.

Van Drie, J., Van Boxtel, C., Jaspers, J., \& Kanselaar, G. (2005). Effects of representational guidance on domain specific reasoning in CSCL. Computers in Human Behavior, 21, $575-602$.

Veldhuis-Diermanse, A. E. (2002). CSCLearning? Participation, learning activities and knowledge construction in computer-supported collaborative learning in higher education. Unpublished Ph.D. dissertation. Wageningen University, The Netherlands. http://www.gcw.nl/dissertations/3187/dis3187.pdf

Vygotsky, L.S. (1978). Interaction between learning and development. En M. Cole, V. JohnSteiner, S. Scribner \& E. Souberman (Eds.), Mind in Society (pp. 79-81). Cambridge, MA: Harvard University Press.

Vygotsky, L.S. (1986). Thought and Language. Cambridge, MA: MIT press.

Wells, G. (1999). Dialogic Inquiry: Towards a Sociocultural Practice and Theory of Education. Cambridge: Cambridge University Press. 
Weinberger, A., Ertl, B., Fischer, F. \& Mandl, H. (2005). Epistemic and social scripts in computer supported collaborative learning. Instructional Science, 33(1), 1-30.

Yin, R. K. (2006). Case Study Methods. En J. L. Green, G. Camilli, \& P. B. Elmore (Eds.), Handbook of Complementary Methods in Education Research. New Jersey: Lawrence Erlbaum Associates Inc. (pp. 111-122). 